PROCEEDINGS OF THE

AMERICAN MATHEMATICAL SOCIETY

Volume 135, Number 11, November 2007, Pages 3703-3709

S 0002-9939(07)08648-0

Article electronically published on August 8, 2007

\title{
LOW FOR RANDOM REALS AND POSITIVE-MEASURE DOMINATION
}

\author{
BJØRN KJOS-HANSSEN
}

(Communicated by Julia Knight)

\begin{abstract}
The low for random reals are characterized topologically, as well as in terms of domination of Turing functionals on a set of positive measure.
\end{abstract}

\section{INTRODUCTION}

A function $f: \omega \rightarrow \omega$ is uniformly almost everywhere (a.e.) dominating if for measure-one many $X$, and all $g$ computable from $X, f$ dominates $g$. Such functions were first studied by Kurtz $[8$ ] who showed that uniformly a.e. dominating functions exist and that in fact $0^{\prime}$, the Turing degree of the halting problem, computes one of them. If we replace measure by category, there are no such functions, as is not hard to see. A few decades later Dobrinen and Simpson [5] made use of a.e. domination in Reverse Mathematics. They made a couple of fundamental conjectures that were promptly refuted in [2] and [4]. In this article we strengthen the results of [2] to provide a characterization of a related concept, positive-measure domination, in terms of lowness for randomness. Conversely, we characterize low for random reals in terms of such domination. The following characterizations are already known. (We assume the reader is familiar with the definition of Martin-Löf random reals and of prefix-free Kolmogorov complexity $K$.)

Theorem 1.1 (Nies, Hirschfeldt, Stephan, Terwijn [6], 10, 11]). The following are equivalent for $A \in 2^{\omega}$ :

- A is low for random: each Martin-Löf random real is Martin-Löf random relative to $A$.

- $A$ is $K$-trivial: $\exists c \forall n K(A\lceil n) \leq K(\emptyset \uparrow n)+c$.

- $A$ is low for $K: \exists c \forall n K(n) \leq K^{A}(n)+c$.

- $\exists Z \geq_{T} A, Z$ is $M L$-random relative to $A$.

- $A \leq_{T} 0^{\prime}$ and $\Omega$ is $M L$-random relative to $A$.

The low for random reals induce a $\Sigma_{3}^{0}$ nonprincipal ideal in the Turing degrees bounded above by a low $\Delta_{2}^{0}$ degree [10, and have already found application to long-standing open problems in computability theory. Our characterizations in

Received by the editors November 29, 2005 and, in revised form, January 21, 2006.

2000 Mathematics Subject Classification. Primary 03D28, 68Q30.

The author thanks the Institute for Mathematical Sciences of the National University of Singapore for support during the preparation of this manuscript at the Computational Prospects of Infinity conference in Summer 2005. The author also thanks Denis R. Hirschfeldt for proving upon request a lemma used in an earlier proof of the case $B \leq_{T} 0^{\prime}$ of Theorem 3.2

(C) 2007 American Mathematical Society Reverts to public domain 28 years from publication 
this paper are distinguished by not being couched in the language of randomness and Kolmogorov complexity. They do however refer to measure; it remains open whether a characterization purely in terms of domination or traces can be given such as that found for low for Schnorr random reals [1], 13].

The first main result of Section 2 is Theorem 2.10, which is a characterization of the low for random reals in terms of containment of effectively closed sets of positive measure. Building on this result, Theorem 2.12 is a characterization of low for random reals in terms of positive-measure domination. Section 3 contains, first, a characterization of the Turing degrees relative to which $0^{\prime}$ is low for random, in terms of positive-measure domination. Finally, with a view toward future research, we include a proof that there is a Turing functional that is universal for this kind of domination.

\section{LOW FOR RANDOM REALS}

To obtain our topological characterization, we will pass first from a certain universal Martin-Löf test (given in terms of $K$ ) to an arbitrary Martin-Löf test, and then to an arbitrary open set of measure $<1$.

Theorem 2.1 (Kraft-Chaitin Theorem [3]). Suppose $\left\langle n_{k}, \sigma_{k}\right\rangle, k \in \omega$, is a recursive sequence, with $\sum_{k} 2^{-n_{k}} \leq 1$. Then there exists a prefix-free machine $M$ and a collection of strings $\tau_{k}$ with $\left|\tau_{k}\right|=n_{k}$ and $M\left(\tau_{k}\right)=\sigma_{k}$.

Definition 2.2 (Chaitin). Let $A \in 2^{\omega}$. An information content measure relative to $A$ is a partial function $\hat{K}: 2^{<\omega} \rightarrow \omega$ such that

$$
\sum_{\sigma \in 2<\omega} 2^{-\hat{K}(\sigma)} \leq 1
$$

and $\{\langle\sigma, k\rangle: \hat{K}(\sigma) \leq k\}$ is r.e. in $A$.

Proof. Let $\sigma_{k}$ be the $k$ th string to enter $V_{n}^{A}$, and let $n_{k}=\left|\sigma_{k}\right|-n$. Note that $\sum_{k} 2^{-n_{k}}=2^{n} \sum_{k} 2^{-\left|\sigma_{k}\right|} \leq 1$, so we can let $M$ be as in Theorem 2.1. In other words, if $\sigma$ enters $V_{n}^{A}$, then produce an $M$-description of $\sigma$ of length $\leq|\sigma|-n$. Let $\hat{K}(\sigma)$ be the length of the shortest description of $\sigma$ so produced.

Lemma 2.3 (Chaitin). If $\hat{K}$ is an information content measure relative to a real $A$, then for all $n, K^{A}(n) \leq \hat{K}(n)+\mathcal{O}(1)$.

Definition 2.4. For any real $X$, let $S^{A}=\left\{S_{n}^{A}\right\}_{n \in \omega}$, where $S_{n}^{A}=\{X: \exists m$ $K^{A}(X\lceil m) \leq m-n\}$.

Lemma 2.5. If $V^{A}$ is a Martin-Löf test relative to $A$, then for each $n$ there exists $p$ such that $V_{p}^{A} \subseteq S_{n}^{A}$.

Proof. For each $m$, write $V_{2 m}^{A}=\bigcup\left\{\left[\sigma_{m, k}\right]: k \in \omega\right\}$ where the function $f$ given by $f(m, k)=\sigma_{m, k}$ is computable and the sets $\left\{\sigma_{m, k}: k \geq 1\right\}$ are prefix-free. Define numbers $n_{m, k}=\left|\sigma_{m, k}\right|-m+1$ for $m, k \in \omega$. We have

$$
\sum_{m, k} 2^{-\left|n_{m, k}\right|}=\sum_{m} 2^{m-1} \sum_{k} 2^{-\left|\sigma_{m, k}\right|}=\sum_{m} 2^{m, 1} \mu V_{2 m} \leq \sum_{m} 2^{m-1} 2^{-2 m}=1 .
$$

Hence by Theorem 2.1 we have a partial $A$-recursive prefix-free machine $M$ and strings $\tau_{m, k}$ with $\left|\tau_{m, k}\right|=n_{m, k}$ and $M\left(\tau_{m, k}\right)=\sigma_{m, k}$. Thus $K_{M}$, complexity based on the machine $M$, satisfies $K_{M}\left(\sigma_{m, k}\right) \leq n_{m, k}$, and so by Lemma 2.3, there is a 
constant $c$ such that $K^{A}\left(\sigma_{m, k}\right) \leq n_{m, k}+c=\left|\sigma_{m, k}\right|-m+1+c$. This means that $V_{2 m}^{A} \subseteq S_{m-c-1}^{A}$ for each $m$.

Thus, given $n$, let $m=n+c+1$ and $p=2 m$. Then $V_{p}^{A}=V_{2 m}^{A} \subseteq S_{m-c-1}^{A}=S_{n}^{A}$, as desired.

Schnorr [12] showed that $S^{A}$ is a universal Martin-Löf test relative to $A$.

Proof. In Lemma 2.5 we can conclude that $\bigcap_{n} V_{n}^{A} \subseteq \bigcap_{n} S_{n}^{A}$. Moreover $\mu S_{n}^{A}=$ $\sum\left\{2^{-|\sigma|}: K(\sigma) \leq|\sigma|-n\right\}<2^{-n}$.

Definition 2.6. Let $n \geq 1$. Let $\Sigma_{n}^{\mu}$ denote the collection of all $\Sigma_{n}^{0}$ classes of measure $<1$. The complement of a $\Sigma_{n}^{\mu}$ class is a $\Pi_{n}^{\mu}$ class. The complement of $U$ is denoted $\bar{U}$. The clopen subset of $2^{\omega}$ generated by $\sigma \in 2^{<\omega}$ is denoted $[\sigma]$, and concatenation of strings is denoted by juxtaposition.

If $U, V$ are open subsets of $2^{\omega}$ given by $U=\bigcup\{[\sigma]: \sigma \in \hat{U}\}$ and $V=\bigcup\{[\sigma]$ : $\sigma \in \hat{V}\}$, where $U$ and $V$ are prefix-free sets of strings, then we define

$$
U V=\bigcup\{[\sigma \tau]: \sigma \in \hat{U}, \tau \in \hat{V}\} .
$$

This product depends on $\hat{U}$ and $\hat{V}$, not just on $U$ and $V$. So when considering a $\Sigma_{1}^{0}$ class $U$, we implicitly fix a suitable recursively enumerable set $\hat{U}$ for $U$.

We define $U^{n}=U^{n-1} U$ where $U^{1}=U$. We can also think of this exponentiation as acting on a closed set $Q$, defining $Q^{n}$ via the equation $\overline{Q^{n}}=\bar{Q}^{n}$. It will be clear whether we are considering a set as open or closed.

Lemma 2.7 (Kučera [7]). For each $\Pi_{1}^{\mu}(A)$ class $Q$ there is a computable function $f$ such that $\left\{\overline{Q^{f(n)}}\right\}_{n \in \omega}$, is a Martin-Löf test relative to $A$.

Proof. Let $q>0$ be a rational number such that $\mu Q \geq q$. Let $P=\bar{Q}$. Then $\mu P^{n}=(\mu P)^{n} \leq(1-q)^{n}$. Let $f$ be a computable function such that for all $k \in \omega$, $\mu P^{f(k)} \leq 2^{-k}$. Let $V_{k}^{A}=P^{f(k)}$. Then $V^{A}$ is a Martin-Löf test relative to $A$.

Lemma 2.8. If $P$ is an open set such that $P^{n}$ is contained in a $\Sigma_{1}^{\mu}$ class for some $n \geq 2$, then $P$ itself is contained in a $\Sigma_{1}^{\mu}$ class.

Proof. We write $U \mid \sigma=\bigcup\{[\tau]:[\sigma \tau] \subseteq U\}$. Note that if $P$ is open, then so is $P^{2}$. Hence by iteration, it suffices to consider the case $n=2$. So suppose $(\exists U) P^{2} \subseteq$ $U \in \Sigma_{1}^{\mu}$. Case 1: $\exists \sigma, \mu(U \mid \sigma)<1, \sigma \in \hat{P}$. Then $P^{2} \cap[\sigma]=[\sigma] P$, the product of $[\sigma]$ and $P$. Then $P=([\sigma] P)\left|\sigma=\left(P^{2} \cap[\sigma]\right)\right| \sigma=P^{2}|\sigma \subseteq U| \sigma \in \Sigma_{1}^{\mu}$. Case 2: Otherwise; so $\hat{P} \subseteq\{\sigma: \mu(U \mid \sigma)=1\}$. Fix $\epsilon>0$ such that $\mu U<1-\epsilon$, and let $V=\bigcup\{[\sigma]: \mu(U \mid \sigma) \geq 1-\epsilon\}$. Note that $V$ is $\Sigma_{1}^{0}$, contains $P$, and $\mu V<1$ because $(1-\epsilon) \mu V \leq \mu U<1-\epsilon$.

As usual, an $A$-random is a real that is Martin-Löf random relative to $A$. If $A, B \in 2^{\omega}$, then $A$ is a tail of $B$ if there exists $n$ such that $A(k)=B(n+k)$ for all $k \in \omega$.

Lemma 2.9 (Kučera [7]). For each $A \in 2^{\omega}$, each $\Pi_{1}^{\mu}(A)$ class contains a tail of each A-random real.

Proof. Let $Q$ be a $\Pi_{1}^{\mu}(A)$ class and suppose $X$ is $A$-random. Then by Lemma 2.7 there is an $m$ such that $X \in Q^{m}$. If $m=2$, then clearly, as $Q$ is closed, some tail of $X$ is an element of $Q$. If $m>2$, the result follows by iteration since each $Q^{m}$ is closed. 
Theorem 2.10. Let $A \in 2^{\omega}$. The following are equivalent:

(1) Each 1-random real is A-random ( $A$ is low for random [10).

(2) For each $\Pi_{1}^{\mu}$ class $Q$ consisting entirely of 1-random reals, there exist $\sigma, n$ such that $Q \cap[\sigma] \neq \emptyset$ but $Q \cap S_{n}^{A} \cap[\sigma]=\emptyset$.

(3) For some $n, \overline{S_{n}^{A}}$ has a $\Pi_{1}^{\mu}$ subclass.

(4) For each A-Martin-Löf test $V_{n}^{A}$, there exists an $n$ such that $\overline{V_{n}^{A}}$ has a $\Pi_{1}^{\mu}$ subclass.

(5) For each $\Pi_{1}^{\mu}(A)$ class $Q$ there exists an $n$ such that $Q^{n}$ has a $\Pi_{1}^{\mu}$ subclass.

(6) Each $\Pi_{1}^{\mu}(A)$ class has a $\Pi_{1}^{\mu}$ subclass.

(7) Some $\Pi_{1}^{\mu}(A)$ class consisting entirely of A-random reals has a $\Pi_{1}^{\mu}$ subclass.

(8) The class of $A$-random reals has a $\Pi_{1}^{\mu}$ subclass.

Proof. $(1) \Rightarrow(2)$ : For this implication we use an argument of Nies and Stephan [9]. Suppose $A$ is low for random but (2) fails. So there is a $\Pi_{1}^{\mu}$ class $Q$ consisting entirely of 1-random reals, such that for all $\sigma, n$, if $Q \cap[\sigma] \neq \emptyset$, then $Q \cap S_{n}^{A} \cap[\sigma] \neq \emptyset$. Let $\sigma_{0}=\lambda$, and $\sigma_{n+1} \succeq \sigma_{n}$, with $\left[\sigma_{n+1}\right] \subseteq S_{n}^{A}$ but $\left[\sigma_{n+1}\right] \cap Q \neq \emptyset$. Then $Y=\bigcup_{n \in \omega} \sigma_{n}$ is not $A$-random, but is 1-random, since $Y \in Q$.

$(2) \Rightarrow(3)$ : Let $Q$ be as in (2), and let $n, \sigma$ be as guaranteed by (2) for $Q$. Then $Q \cap[\sigma]$ is the desired subclass. It has positive measure because no 1-random belongs to a $\Pi_{1}^{0}$ class of measure zero.

$(3) \Rightarrow(4)$ : Lemma 2.5 .

$(4) \Rightarrow(5)$ : Let $Q$ be a $\Pi_{1}^{\mu}(A)$ class. By Lemma $2.7 V_{k}^{A}=\overline{Q^{f(k)}}$ is a Martin-Löf test relative to $A$ for some computable $f$. By $(4), Q^{f(m)}=\overline{V_{m}^{A}} \supseteq F$ for some $F \in \Pi_{1}^{\mu}$ and $m$; let $n=f(m)$.

$(5) \Rightarrow(6)$ : Lemma 2.8 .

$(6) \Rightarrow(7)$ : If $U^{A}$ is a universal Martin-Löf test for $A$-randomness, then we can let $Q=\overline{U_{1}}$.

$(7) \Rightarrow(8)$ : Since any class consisting entirely of $A$-randoms is contained in the class of all $A$-randoms.

$(8) \Rightarrow(1)$ : Suppose $X$ is 1-random; we need to show $X$ is $A$-random. Let $F$ be a $\Pi_{1}^{\mu}$ subclass of the class of $A$-randoms. By Lemma 2.9, some tail of $X$ is an element of $F$. Hence a tail of $X$ is $A$-random, and thus $X$ itself is $A$-random.

To characterize the low for random reals in terms of domination we first introduce some notation. We write

$$
\operatorname{Tot}(\Phi)=\left\{X: \Phi^{X} \text { is total }\right\} \quad \text { and } \quad \varphi^{X}(n)=(\mu s)(\forall m<n)\left(\Phi_{s}^{X}(m) \downarrow \leq s\right) .
$$

Note that $\operatorname{Tot}(\Phi)$ is a $\Pi_{2}^{0}$ class for each $\Phi$, and $\operatorname{Tot}(\Phi)=\operatorname{Tot}(\varphi)$. The function $\varphi$ is the running time of $\Phi$, explicitly satisfying $\Phi^{X}(n) \leq \varphi^{X}(n)$ for all $n$. Let $\Phi$ be a Turing functional and $B \in 2^{\omega}$. If there exists $f \leq_{T} B$ such that for positivemeasure many $X, \Phi^{X}$ is dominated by $f$, then we write $\Phi<B$. By $\sigma$-additivity this is equivalent to the statement that there exists $f \leq_{T} B$ such that for positivemeasure many $X, \Phi^{X}$ is majorized by $f$. We also write $\Phi<B$ in the case that $\operatorname{Tot}(\Phi)$ has measure zero.

Lemma 2.11 (implicit in [5]). Let $B \in 2^{\omega}$ and let $\Phi$ be a Turing functional. Then $\varphi<B$ iff $\operatorname{Tot}(\Phi)$ has a $\Pi_{1}^{\mu}(B)$ subclass. 
Proof. First suppose $\varphi<B$, as witnessed by $f$. Then $\left\{X: \forall n \Phi_{f(n)}^{X}(n) \downarrow\right\}$ is a $\Pi_{1}^{\mu}(B)$ subclass of $\operatorname{Tot}(\Phi)$. Conversely, let $F$ be a $\Pi_{1}^{\mu}(B)$ subclass of $\operatorname{Tot}(\Phi)$. By compactness, $\left\{\varphi^{X}(n): X \in F\right\}$ is finite for each $n$, and $\{\langle n, m\rangle: \forall X(X \in$ $\left.F \rightarrow \varphi^{X}(n)<m\right\}$ is a $\Sigma_{1}^{0}(B)$ class. Hence by $\Sigma_{1}^{0}(B)$ uniformization there is a function $f \leq_{T} B$ such that $\forall n \forall X\left(X \in F \rightarrow \varphi^{X}(n)<f(n)\right)$; i.e., $f$ witnesses that $\varphi<B$.

Theorem 2.12. Let $A \in 2^{\omega}$. The following are equivalent:

(1) $A$ is low for random.

(2) Each $\Pi_{1}^{\mu}(A)$ class has a $\Pi_{1}^{\mu}$ subclass.

(3) (i) $A \leq_{T} 0^{\prime}$, and (ii) for each $\Phi$, if $\operatorname{Tot}(\Phi)$ has a $\Pi_{1}^{\mu}(A)$ subclass, then $\varphi<0$.

(4) (i) $A \leq_{T} 0^{\prime}$, and (ii) for each $\Phi$, if $\varphi<A$, then $\varphi<0$.

Proof. $(1) \Leftrightarrow(2)$ was shown in Theorem 2.10 ,

$(2) \Rightarrow(3)$ : Nies 10 shows that if $A$ is low for random, then $A \leq_{T} 0^{\prime}$. Suppose $\operatorname{Tot}(\Phi)$ has a $\Pi_{1}^{\mu}(A)$ subclass $Q$. By $(2), \operatorname{Tot}(\Phi)$ has a $\Pi_{1}^{\mu}$ subclass $F$. By Lemma 2.11, we are done.

$(3) \Rightarrow(2)$ : Suppose $(3)$ holds and suppose $Q$ is a $\Pi_{1}^{\mu}(A)$ class. Pick $\Psi$ such that $Q=\left\{X: \Psi^{X \oplus A}(0) \uparrow\right\}$. Since $A \leq_{T} 0^{\prime}, A=\lim _{s} A_{s}$, the limit of a computable approximation. Let $\Phi^{X}(s)=\mu t>s\left(\Psi_{t}^{X \oplus A_{t}}(0) \uparrow\right)$. Then $Q=\operatorname{Tot}(\Phi)$. Applying (3) to this $\Phi$, we have $\varphi<0$, and so by Lemma 2.11 we are done.

$(3) \Leftrightarrow(4)$ is immediate from Lemma 2.11

\section{Positive-measure domination}

In [5] it was asked whether the Turing degrees $A$ of uniformly a.e. dominating functions are characterized by either of the inequalities $A \geq 0^{\prime}$ and $A^{\prime} \geq_{T} 0^{\prime \prime}$. The case $A \geq 0^{\prime}$ was refuted by a direct construction in $\left[4\right.$. The case $A^{\prime} \geq_{T} 0^{\prime \prime}$ was refuted in [2] using precursors to the results presented here. Namely, the dual of property 4 (ii) above is $\forall \varphi(\varphi<A)$ or equivalently $\forall \varphi\left(\varphi<0^{\prime} \rightarrow \varphi<A\right)$. Relativizing our proofs gives that this is equivalent to: $0^{\prime}$ is low for random relative to $A$. If we restrict ourselves to $A \leq_{T} 0^{\prime}$, then by [10] this implies $A^{\prime} \geq_{t t} 0^{\prime \prime}$, which is strictly stronger than $A^{\prime} \geq_{T} 0^{\prime \prime}$. We do not know whether the assumption $A \leq_{T} 0^{\prime}$ is necessary for either of the conclusions $A^{\prime} \geq_{t t} 0^{\prime \prime}, A^{\prime} \geq_{T} 0^{\prime \prime}$.

We say that $A$ is positive-measure dominating if for each $\Phi, \Phi<A$. If each $B$-random real is $A$-random, then we write $A \leq_{L R} B$ (A is low for random relative to $B$ ) following [10]. We write $\Phi^{A}$ for the functional $X \mapsto \Phi^{A \oplus X}$.

Lemma 3.1. Let $A \in 2^{\omega}$ and $\mathcal{C} \subseteq 2^{\omega}$. Then $\mathcal{C}$ is a $\Pi_{2}^{0}(A)$ class iff $\mathcal{C}$ is $\operatorname{Tot}\left(\Phi^{A}\right)$ for some Turing functional $\Phi$.

Proof. Suppose $\mathcal{C}$ is a $\Pi_{2}^{0}(A)$ class, i.e. $\mathcal{C}=\{X: \forall y \exists s R(y, s, A, X)\}$, where $R$ is a formula in the language of second-order arithmetic all of whose quantifiers are firstorder and bounded. Then we can let $\Phi^{A \oplus X}(y)=\mu s(R(y, s, A, X))$. Conversely, $\operatorname{Tot}\left(\Phi^{A}\right)=\left\{X: \forall y \exists s\left(\Phi_{s}^{A \oplus X}(y) \downarrow\right)\right\}$.

Theorem 3.2. Let $B \in 2^{\omega}$. Then $0^{\prime}$ is low for random relative to $B$ iff $B$ is positive-measure dominating. 
Proof. This is the special case $A=0$ of the fact that for each $A, B \in 2^{\omega}$, the following are equivalent:

(1) $A^{\prime} \leq_{L R} A \oplus B$.

(2) Each $\Pi_{1}^{\mu}\left(A^{\prime}\right)$ class has a $\Pi_{1}^{\mu}(A \oplus B)$ subclass.

(3) Each $\Pi_{2}^{\mu}(A)$ class has a $\Pi_{1}^{\mu}(A \oplus B)$ subclass.

(4) $\forall \Phi$, if $\operatorname{Tot}\left(\Phi^{A}\right)$ has positive measure, then it has a $\Pi_{1}^{\mu}(A \oplus B)$ subclass.

(5) $\forall \Phi\left(\varphi^{A}<A \oplus B\right)$.

The equivalences are proved as follows. $(1) \Leftrightarrow(2)$ : Relativization of Theorem 2.10 gives: $A \leq_{L R} B$ iff each $\Pi_{1}^{\mu}(A)$ class has a $\Pi_{1}^{\mu}(B)$ subclass. $(3) \Leftrightarrow(4)$ : Lemma 3.1 $(4) \Leftrightarrow(5)$ : Relativization of Lemma 2.11 $(2) \Leftrightarrow(3)$ : Let $A \in 2^{\omega} . A^{\prime}$ is uniformly a.e. dominating relative to $A$, hence $A^{\prime}$ is positive-measure dominating relative to $A$. Hence by putting $B=A^{\prime}$ in $(3) \Leftrightarrow(5)$, each $\Pi_{2}^{\mu}(A)$ class has a $\Pi_{1}^{\mu}\left(A^{\prime}\right)$ subclass.

Universal functionals. Suppose $\Phi_{i}, i \in \omega$, are all the Turing functionals. As observed in [4, the functional $\Psi$ given by $\Psi^{0^{i} 1 X}=\Phi_{i}^{X}$ is universal for uniform a.e. domination, in the sense that any function that dominates $\Psi$ on almost every $X$ is a uniformly a.e. dominating function. As $\Psi<0, \Psi$ is not universal for positive-measure domination; however, the following functional is universal.

Fix $c \in \omega$. Let $\Xi_{c}^{X}(s)$ be the least stage $t>s$ at which $X$ looks like it is 2-random, with constant $c$. That is,

$$
\Xi_{c}^{X}(s)=(\mu t>s)\left(\forall n \leq t K_{t}^{0_{t}^{\prime}}(X\lceil n) \geq n-c) .\right.
$$

Here $0_{t}^{\prime}$ is the approximation to $0^{\prime}$ at stage $t$, and $K_{t}^{A}$ the approximation to $K^{A}$ for any $A \in 2^{\omega}$. Considering $S_{c}^{X}$ (Definition 2.4), it is clear that $\Xi_{c}$ is total for positive-measure many $X$, all of which are 2-randoms. The running time $\xi_{c}$ of $\Xi_{c}$ is universal for positive-measure domination in the following sense.

Theorem 3.3. The class $\{A: A$ is positive-measure dominating $\}$ is $\Sigma_{3}^{0}$. In fact, for each $A \in 2^{\omega}$ and $c \in \omega, A$ is positive-measure dominating iff $\xi_{c}<A$.

Proof. The complement of $\operatorname{Tot}\left(\Xi_{c}\right)$ is $\left\{X: \exists n K^{0^{\prime}}(X\lceil n)<n-c\}\right.$, which is open. Hence $\operatorname{Tot}\left(\Xi_{c}\right)$ is closed and is in fact a $\Pi_{1}^{\mu}\left(0^{\prime}\right)$ class. Suppose $\xi_{c}<A$. By Lemma 2.11, $\operatorname{Tot}\left(\Xi_{c}\right)$ has a $\Pi_{1}^{\mu}(A)$ subclass. Thus: Some $\Pi_{1}^{\mu}\left(0^{\prime}\right)$ class consisting entirely of $0^{\prime}$-randoms has a $\Pi_{1}^{\mu}(A)$ subclass. By Theorem 2.10(7) relativized, $0^{\prime} \leq_{L R} A$, and so by Theorem 3.2. $A$ is positive-measure dominating.

\section{REFERENCES}

1. A. Nies, B. Kjos-Hanssen and F. Stephan, Lowness for the class of Schnorr random reals, SIAM J. Computing 35 (2006), no. 3, 647-657. MR2201451

2. S. Binns, B. Kjos-Hanssen, M. Lerman, and D.R. Solomon, On a question of Dobrinen and Simpson concerning almost everywhere domination, J. Symbolic Logic 71 (2006), no. 1, 119136.

3. G.J. Chaitin, A theory of program size formally identical to information theory, J. Assoc. Comput. Mach. 22 (1975), 329-340. MR0411829 (53:15557)

4. P. Cholak, N. Greenberg, and J.S. Miller, Uniform almost everywhere domination, J. Symbolic Logic 71 (2006), no. 3, 1057-1072. MR2251556

5. N.L. Dobrinen and S.G. Simpson, Almost everywhere domination, J. Symbolic Logic 69 (2004), 914-922. MR2078930(2005d:03079)

6. D.R. Hirschfeldt, A. Nies, and F. Stephan, Using random sets as oracles, to appear. 
7. Antonín Kučera, Measure, $\Pi_{1}^{0}$-classes and complete extensions of PA, Recursion theory week (Oberwolfach, 1984), Lecture Notes in Math., vol. 1141, Springer, Berlin, 1985, pp. 245-259. MR 0820784 (87e:03102)

8. S.A. Kurtz, Randomness and genericity in the degrees of unsolvability, Ph.D. thesis, University of Illinois at Urbana-Champaign, 1981, pp. VII+131 pages.

9. A. Nies, Low for random reals: the story, unpublished.

10. L Lowness properties and randomness, Adv. Math. 197 (2005), 274-305. MR2166184

11. A. Nies, F. Stephan, and S.A. Terwijn, Randomness, relativization and Turing degrees, J. Symbolic Logic 70 (2005), no. 2, 515-535. MR2140044

12. C.P. Schnorr, A unified approach to the definition of a random sequence, Mathematical Systems Theory 5 (1971), 246-258. MR0354328 (50:6808)

13. S.A. Terwijn and D. Zambella, Computational randomness and lowness, J. Symbolic Logic 66 (2001), 1199-1205. MR.1856736 (2002j:03044)

Department of Mathematics, Cornell University, Ithaca, New York 14853

E-mail address: bjoern@math.cornell.edu 